guidelines for staff, teaching sessions and the development of a standardised protocol for timely report to GP.

\section{P-121 SERVICE EVALUATION: WHAT HAPPENED TO HOSPICE IN-PATIENTS TRANSFERRED TO AN ACUTE HOSPITAL AND LESSONS LEARNED}

\author{
1,2Patricia Strubbe, ${ }^{2,3}$ Katrien Naessens. 'Sue Ryder Duchess of Kent Hospice, Reading, UK; \\ ${ }^{2}$ Sue Ryder, Reading, UK; ${ }^{3}$ Royal Berkshire Hospital Foundation Trust
}

10.1136/bmjspcare-2017-00133.120

The 2 hospice in-patient units combined have 27 in-patient beds. For every admission we document decisions about cardio-pulmonary resuscitation and transfer to acute hospital. Both units offer intra-venous treatments (blood products, bisphosphonates and antibiotics). Previously published audits ${ }^{1}$ were done in units were intravenous antibiotics were not available. This retrospective service evaluation was done in order to evaluate what happened to transferred patients and whether we can improve our practice in the future.

The authors looked at the clinical notes of all transfers to acute hospital between January 2014 and July 2015. Case finding relied on memory and documentation in admission books.

There were 16 transfer (involving 13 patients) 8 for diagnosis (fracture, MSCC, PE) 7 for treatment (electrolyte abnormality, neutropaenic sepsis and NIV initiation) and 1 for a post-surgical complication. Ten transfers happened during normal working hours and six out of usual working hours. Decisions tended to be well documented and consultants were involved in eleven cases.

In 11 instances the patient returned to the unit, 2 died in hospital, 2 were discharged home, 1 patient died 3 weeks later (location unclear) and in 12 cases the aim of the transfer was met.

Further analysis revealed that most transfer decisions were well documented. However what information was sent with the patient and criteria for return to the unit were not clear and patients lingered longer than intended in the acute hospital. We did not evaluate decisions not to transfer to hospital.

In future we aim

- Not only to document suitability for transfer on admission but also review this regularly.

- To document changes in clinical condition which may lead to transfer to acute hospital whether or not patient is transferred.

- On transfer to communicate doctor-to-doctor with clear goals and return criteria

- To liaise with hospital palliative care team.

\section{REFERENCES}

1. Castanheira T, Wright BA. Survey about patients transferred from a Specialist Palliative Care unit to an acute hospital setting . European Journal of Palliative Care 2014;21:219-221

2. Doidge $M$, Perkins P. Letter to the editor. European Journal of Palliative Care 2015;22(1)

\section{P-122 LIDOCAINE 5\% PATCH INITIATION AND ASSESSMENT}

Kirsty Lowe. Roxburghe House, Royal Victoria Hospital, Dundee, UK, DD2 1SP
Background The Scottish Palliative Care Guidelines (SPCG) provide guidance for initiating lidocaine $5 \%$ patches and assessing their efficacy. As most patients will respond within 1-2 weeks, patches should be reviewed around this time, by way of a patch-free trial, and discontinued if not beneficial.

In Roxburghe House hospice, Dundee, lidocaine patches are not routinely reviewed. My aim was to educate prescribers regarding national guidance, and to encourage them to review lidocaine patches 1-2 weeks after initiation.

Methods A record was kept of all inpatients who commenced lidocaine patches in Roxburghe between $1^{\text {st }}$ December 2015 and $29^{\text {th }}$ February 2016. Indication for starting patch, pain assessments and outcome of patch-free trial were all recorded.

Following cycle 1 I provided education sessions about the SPCG guidance for prescribers in Roxburghe. I introduced an assessment sheet based on the SPCG recommendations. I then repeated the data collection with patients who commenced lidocaine patches in Roxburghe House between $1^{\text {st }}$ July and $30^{\text {th }}$ September 2016.

Results Cycle 1 confirmed that the SPCG guidance was not being followed in Roxburghe House. Only one patient had a patch-free trial and the majority of patients had no pain assessment at 48 hours or 1-2 weeks.

In cycle $2100 \%$ of patients had pain scores documented, although only $50 \%$ had the new assessment form completed. Of the patients who survived past 1-2 weeks, $100 \%$ had clear instructions to their GP requesting review of the patch in the community.

Conclusions The introduction of a lidocaine patch assessment form has had some success so far in Roxburghe House, however further education is required to reinforce the importance of following the SPCG guidance. Other interventions which may be interesting would be providing education updates on the SPCG guidance for GPs and formulating an information leaflet for patients which provides instructions for how and when to initiate a patch-free trial.

\section{P-123 USING THE MODEL OF IMPROVEMENT TO INCREASE THE EFFICIENCY OF DISCHARGE MEDICATION PRESCRIBING IN PALLIATIVE CARE}

Rory Carrigan, Charles Daniels. St. Luke's Hospice (Harrow and Brent), Harrow, UK

\subsection{6/bmjspcare-2017-00133.122}

Aim To reduce the time taken and the quality of To Take Away (TTA) prescribing in a hospice setting

Background Using and learning from the model for improvement can help guide us through change. A hospice discharge process can be lengthy and complicated by the prescription of medications. It was hypothesised that too much time was being spent by clinicians on handwriting discharge prescriptions (FP10s). We proposed that typing, printing and electronically storing FP10 prescriptions would reduce the time taken and improve legibility.

Method An electronic TTA template was created in MS Word and stored on a secured network. We measured the time taken to produce and process handwritten and electronic TTAs after a period of change. The whole process was timed from creation to electronic submission to the pharmacy. Any enquiries or extra work sought by the pharmacy team was measured as an additional added time to this process. 